\title{
The Spatio-Temporal Impulse Response of the Linearized Navier-Stokes Equations ${ }^{1}$
}

\author{
Mihailo Jovanović \\ jmihailo@engineering.ucsb.edu
}

\author{
Bassam Bamieh \\ bamieh@engineering.ucsb.edu
}

Department of Mechanical and Environmental Engineering
University of California, Santa Barbara, CA 93106-5070

\begin{abstract}
In this paper, we perform an input-output analysis of the linearized Navier-Stokes equations by investigating their spatio-temporal 'impulse response'. Our first motivation for this problem comes from experimental work, where transition to turbulence is observed to occur either naturally, with so-called 'turbulent spots', or when these spots are intentionally induced in the flow by deliberate external excitation, which can be modeled as a spatio-temporal impulse body force in the Navier-Stokes equations. We compare this computed spatio-temporal impulse response to observed characteristics of turbulent spots and find significant qualitative agreement. Our other motivation is to fully probe the dynamics of the linearized equations, and we find that they exhibit rich and complex structures hitherto unseen by other analysis methods.
\end{abstract}

\section{Introduction}

One of the main issues in the theory of hydrodynamic stability for fluid flows is to determine the structure of flow perturbations that lead to transition to turbulence. The classical approach to this problem involves the spectral (i.e. normal mode) analysis of the Navier-Stokes equations linearized around certain laminar flow condition. For low Reynolds numbers all flows are stable, and classical analysis is thus concerned with finding the critical Reynolds numbers at which unstable normal modes appear, or equivalently, when the generator of the evolution equation becomes unstable. For wall bounded shear flows, this analysis leads to transition theories based on the well known Tollmien-Schlichting waves, which have long been considered to be the primary mechanism of instability leading to transition. However, this theory is quite unsatisfactory for describing experimentally observed phenomena in so-called 'natural transition'. In such cases, the transition Reynolds numbers, as well as the dominant flow structures, are not related to Tollmien-Schlichting waves.

\footnotetext{
${ }^{1}$ Research supported in part by NSF under Grant ECS96-24152.
}

It has become clear from recent work $[1,2,3,4,5]$ that this long-standing difficulty with classical linear hydrodynamic stability theory is overcome by a different analysis of the linearized Navier-Stokes equations. It turns out that these equations represent a system, though nominally stable (at most Reynolds numbers), which is extremely sensitive to external excitations and unmodeled dynamics. For instance, despite the fact that the equations represent a stable evolution (for e.g. pipe flows, or Poiseuille flow for Reynolds numbers below 5772), the margin of stability of a robust stability problem is very small, and decreases with Reynolds number. Furthermore, under external excitation (which is arguably present in all physical flow problems), disturbances are amplified significantly. Perhaps the most dramatic difference between this analysis and the classical one is captured in the fact that under external excitations, the input-output resonances of the equations occur at very different spatio-temporal frequencies than the poorly damped modes of the system. These poorly damped modes represent TollmienSchlichting waves, while the input-output resonances are related to the streamwise vortices and streaks, which are ubiquitous in transitioning shear flows and fully turbulent boundary layers.

In this paper, we carry the input-output analysis further by investigating the 'impulse response' of the linearized Navier-Stokes equations. Our motivation for this problem comes from experimental work where transition is observed to occur either naturally, beginning with so-called 'turbulent spots', or when these spots are intentionally induced in the flow by deliberate external excitation. This external excitation can often be modeled as a spatio-temporal impulse occurring as a body force in the Navier-Stokes equations. Our intention is to compare the spatio-temporal impulse response of the linearized Navier-Stokes equations with observed characteristics of turbulent spots.

Another motivation for considering a spatiotemporal impulse response is the need to fully probe the dynamics of the linearized Navier-Stokes equations. These equations have recently been 
shown to have richer and more complex dynamical behavior than previously thought. Other analysis methods, such as normal modes or eigenfunction expansions, typically do not show the full range of behaviors of these equations. A spatio-temporal impulse response appears to be an effective analysis method, since all modes of the system are excited simultaneously. This intuition appears to be justified by our results, which show that the impulse response exhibits complex flow structures hitherto unseen in any other linear analysis.

Our methodology involves deriving an evolution model where the linearized Navier-Stokes equations are subject to body forces that model external excitations. These excitations represent several influences such as wall roughness, free stream turbulence and/or neglected nonlinearities. The underlying equations are partial differential equations in three dimensions. We investigate the impulse response by exploiting the spatial invariance of the equations in two of the dimensions (the so-called streamwise and spanwise directions), and numerically approximating the PDE in the remaining wall-normal direction. The first part of the paper is devoted to deriving the input-output model, the impulse response computations and the numerical schemes used. In the remainder, we present the numerical results, compare the computed flow fields with experimental observations, and make some conclusions about the utility of the impulse response in understanding the behavior of distributed parameter systems.

\section{Linearized Navier-Stokes Equations Subject to External Forces}

A three dimensional channel flow between two parallel infinite plates is considered (See Figure 1 for geometry). In the case of incompressible flow, with a nominal velocity profile $U_{m}(x, y, z)=U(y)$, $-1 \leq y \leq 1$ the perturbations of the velocity and pressure fields are to satisfy the linearized NavierStokes equations

$$
\begin{array}{ll}
\partial_{t} u+U \partial_{x} u+U^{\prime} v & =-\partial_{x} p+\frac{1}{R} \Delta u, \\
\partial_{t} v+U \partial_{x} v & =-\partial_{y} p+\frac{1}{R} \Delta v, \\
\partial_{t} w+U \partial_{x} w & =-\partial_{z} p+\frac{1}{R} \Delta w,
\end{array}
$$

and the continuity equation

$$
\partial_{x} u+\partial_{y} v+\partial_{z} w=0 .
$$

All velocities and lengths are non-dimensionalized by the maximal velocity $U_{0}$ and the half-width of the channel $L$, respectively. These two quantities, together with the kinematic viscosity $\nu$ determine the value of the Reynolds number, $R:=\frac{U_{0} L}{\nu}$. The three dimensional Laplacian is denoted by $\Delta:=$

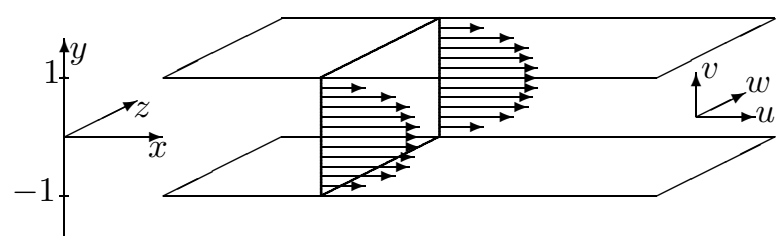

Figure 1: Three dimensional channel flow.

$\partial_{x x}^{2}+\partial_{y y}^{2}+\partial_{z z}^{2}$, and $U^{\prime}:=\partial_{y} U$. Each field is assumed to be both temporally and spatially varying, e.g. $p=p(x, y, z, t)$. The linearized Navier-Stokes equations can be also considered in a slightly different way. The mathematical representation given by (1) is derived based on the assumption that no external body forces are present. This assumption is hardly plausible in the real setting. Different kinds of disturbances always accompany any fluid flow. Let us mention some of them: surface irregularities, free stream disturbances, acoustics, vibrations of the wind tunnel walls, etc. In order to include their influence, additional terms can be added on the right hand side of the equation (1)

$$
\begin{aligned}
\partial_{t} u+U \partial_{x} u+U^{\prime} v & =-\partial_{x} p+\frac{1}{R} \Delta u+F_{u}, \\
\partial_{t} v+U \partial_{x} v & =-\partial_{y} p+\frac{1}{R} \Delta v+F_{v}, \\
\partial_{t} w+U \partial_{x} w & =-\partial_{z} p+\frac{1}{R} \Delta w+F_{w} .
\end{aligned}
$$

These terms can account for the neglected nonlinearities, as well.

One can rewrite the last equations as

$\partial_{t}\left[\begin{array}{c}u \\ v \\ w\end{array}\right]=\overline{\mathcal{A}}\left[\begin{array}{c}u \\ v \\ w\end{array}\right]-\left[\begin{array}{c}\partial_{x} \\ \partial_{y} \\ \partial_{z}\end{array}\right] p+\left[\begin{array}{c}F_{u} \\ F_{v} \\ F_{w}\end{array}\right]$,

and think of them as a dynamical system with a forcing term $F:=\left[\begin{array}{lll}F_{u} & F_{v} & F_{w}\end{array}\right]^{\prime}$ and an input $p$. The latter has to adjust itself in order for the continuity equation to be satisfied. Equation (2) implies that the state of the system (3) evolves in a divergence free linear subspace determined by $\mathcal{N}(\operatorname{div})$, where $\mathcal{N}(\cdot)$ denotes the null space of a certain operator. Hence, the state of the system under consideration can be parametrized by only two fields. Clearly, this parameterization may be done in a number of different ways. A choice that is particularly suitable is given in terms of the wall normal velocity and vorticity fields

$$
\psi:=\left[\begin{array}{c}
v \\
\omega_{y}
\end{array}\right]=\left[\begin{array}{ccc}
0 & I & 0 \\
\partial_{z} & 0 & -\partial_{x}
\end{array}\right]\left[\begin{array}{c}
u \\
v \\
w
\end{array}\right] .
$$

By combining (3) and (2) we can express pressure as a function of $v, U^{\prime}, F_{u}, F_{v}$ and $F_{w}$

$$
\Delta p=-2 U^{\prime} \partial_{x} v+\partial_{x} F_{u}+\partial_{y} F_{v}+\partial_{z} F_{w},
$$


while by applying the Laplacian to the second row of (3) we obtain

$$
\Delta \partial_{t} v=-\Delta\left(U \partial_{x} v\right)-\partial_{y}(\Delta p)+\frac{1}{R} \Delta^{2} v+\Delta F_{v} .
$$

A single equation for the wall normal velocity $v$ is obtained by substituting (5) into (6)

$$
\begin{aligned}
\Delta \partial_{t} v= & -U \partial_{x} \Delta v+U^{\prime \prime} \partial_{x} v+\frac{1}{R} \Delta^{2} v \\
& -\partial_{x y}^{2} F_{u}+\left(\partial_{x x}^{2}+\partial_{z z}^{2}\right) F_{v}-\partial_{y z}^{2} F_{w},
\end{aligned}
$$

while a single equation for its wall normal vorticity counterpart is obtained by subtracting the partial derivative of the third row of (3) with respect to $x$ from $\partial_{z}$ of the first row of the same equation

$$
\partial_{t} \omega_{y}=-U^{\prime} \partial_{z} v-U \partial_{x} \omega_{y}+\frac{1}{R} \Delta \omega_{y}+\partial_{z} F_{u}-\partial_{x} F_{w} .
$$

In that way we are able to convert the model expressed by (3) and (2) into a more suitable form

$$
\begin{aligned}
\partial_{t}\left[\begin{array}{c}
v \\
\omega_{y}
\end{array}\right] & =\left[\begin{array}{cc}
\mathcal{L} & 0 \\
\mathcal{C} & \mathcal{S}
\end{array}\right]\left[\begin{array}{c}
v \\
\omega_{y}
\end{array}\right]+\mathcal{B} F \\
& =: \mathcal{A} \psi+\mathcal{B} F
\end{aligned}
$$

where

$$
\begin{aligned}
\mathcal{L} & :=\Delta^{-1}\left(-U \partial_{x} \Delta+U^{\prime \prime} \partial_{x}+\frac{1}{R} \Delta^{2}\right), \\
\mathcal{S} & :=-U \partial_{x}+\frac{1}{R} \Delta, \\
\mathcal{C} & :=-U^{\prime} \partial_{z},
\end{aligned}
$$

and

$$
\mathcal{B}:=\left[\begin{array}{cc}
\Delta^{-1} & 0 \\
0 & I
\end{array}\right]\left[\begin{array}{ccc}
-\partial_{x y}^{2} & \partial_{x x}^{2}+\partial_{z z}^{2} & -\partial_{y z}^{2} \\
\partial_{z} & 0 & -\partial_{x}
\end{array}\right] .
$$

A great advantage of the mathematical representation given by equation $(7)^{1}$ is that the only additional constraints that the wall normal velocity and vorticity fields have to obey are the boundary conditions

$$
\begin{aligned}
& v(x, \pm 1, z, t)=\partial_{y} v(x, \pm 1, z, t)=0 \\
& \omega_{y}(x, \pm 1, z, t)=0, \quad \forall x, z, t \in \mathbb{R} .
\end{aligned}
$$

These constraints follow from the no-slip boundary conditions on the original velocity fields and the continuity equation.

The operators $\mathcal{L}$ and $\mathcal{S}$ are called the Orr-Sommerfeld and Squire operators, respectively, while the operator $\mathcal{C}$ represents the coupling from the wall normal velocity to the wall normal vorticity.

A closer look at the model in the evolution form reveals that the generator $\mathcal{A}$ is translation invariant in the $x$ and $z$ directions (but not in $y$ !). This fact allows us to find the Fourier transforms of the wall normal velocity and vorticity fields ${ }^{2}$

$$
\hat{\psi}\left(k_{x}, y, k_{z}, t\right):=\int_{\mathbb{R}} \int_{\mathbb{R}} \psi(x, y, z, t) e^{-i\left(x k_{x}+z k_{z}\right)} d x d z,
$$

where $i$ is the imaginary unit, while $k_{x}$ and $k_{z}$ are the spatial frequencies or the so-called wave-numbers in the streamwise and spanwise directions. In this way, the

\footnotetext{
${ }^{1}$ The model given by this equation will be referred to as the model in the evolution form.

${ }^{2} \mathrm{As}$ well as the Fourier transform of the forcing term.
}

model given by equation (7) simplifies to the one dimensional PDE parametrized by $k_{x}$ and $k_{z}$

$$
\begin{aligned}
\partial_{t}\left[\begin{array}{c}
\hat{v} \\
\hat{\omega}_{y}
\end{array}\right] & =\left[\begin{array}{cc}
\hat{\mathcal{L}} & 0 \\
\hat{\mathcal{C}} & \hat{\mathcal{S}}
\end{array}\right]\left[\begin{array}{c}
\hat{v} \\
\hat{\omega}_{y}
\end{array}\right]+\hat{\mathcal{B}} \hat{F} \\
& =: \hat{\mathcal{A}} \hat{\psi}+\hat{\mathcal{B}} \hat{F}
\end{aligned}
$$

where $\hat{\mathcal{L}}, \hat{\mathcal{S}}$, and $\hat{\mathcal{C}}$ represent the Fourier transforms of the operators defined in (8)

$$
\begin{aligned}
\hat{\mathcal{L}} & :=\Delta^{-1}\left(-i k_{x} U \Delta+i k_{x} U^{\prime \prime}+\frac{1}{R} \Delta^{2}\right), \\
\hat{\mathcal{S}} & :=-i k_{x} U+\frac{1}{R} \Delta, \\
\hat{\mathcal{C}} & :=-i k_{z} U^{\prime} .
\end{aligned}
$$

Note that the Laplacian, once the Fourier transform is applied, becomes equal to $\Delta=\partial_{y y}^{2}-k_{x}^{2}-k_{z}^{2}$.

\section{Spatio-Temporal Impulse Response of the Linearized Navier-Stokes Equations}

Our ultimate goal in this section is to compute the response of the system whose mathematical model can be represented by (7). We assume the forcing of the form

$$
F_{u}=F_{w}=0, \quad F_{v} \approx \delta(x, y+1-\varepsilon, z, t),
$$

where $\delta(x, y+1-\varepsilon, z, t)$ is the Dirac delta function and $0<\varepsilon \ll 1$. This means that we want to introduce an input in the form of a spatio-temporal impulse function, located near the lower wall, that enters only into the equation governing wall normal velocity component. This particular choice is appealing because it should help us get a better understanding of how changes in $v$ influence, through the coupling term $\mathcal{C}$, the evolution of $\omega_{y}[5]$.

Based on our choice of the forcing term one rewrites (9) as

$\partial_{t} \hat{\psi}=\left[\begin{array}{cc}\hat{\mathcal{L}} & 0 \\ \hat{\mathcal{C}} & \hat{\mathcal{S}}\end{array}\right] \hat{\psi}+\left[\begin{array}{c}-\Delta^{-1}\left(k_{x}^{2}+k_{z}^{2}\right) \\ 0\end{array}\right] \hat{F}_{v}$,

where

$$
\begin{aligned}
\hat{F}_{v}\left(k_{x}, y, k_{z}, t\right) & =\int_{\mathbb{R}} \int_{\mathbb{R}} F_{v}(x, y, z, t) e^{-i\left(x k_{x}+z k_{z}\right)} d x d z \\
& =\delta(y+1-\varepsilon, t),
\end{aligned}
$$

with the boundary conditions on $\hat{v}$ and $\hat{\omega}_{y}$ given by

$$
\begin{aligned}
& \hat{v}\left(k_{x}, \pm 1, k_{z}, t\right)=\partial_{y} \hat{v}\left(k_{x}, \pm 1, k_{z}, t\right)=0, \\
& \hat{\omega}_{y}\left(k_{x}, \pm 1, k_{z}, t\right)=0, \quad \forall k_{x}, k_{z}, t \in \mathbb{R} .
\end{aligned}
$$

Subsection 3.1 describes, in detail, the numerical procedure used for solving equation (10) subject to the previously described forcing term, and the boundary conditions given by (11). Subsection 3.2 contains the description of simulation results obtained by computing the impulse response of the linearized Navier-Stokes equations in the case of Poiseuille flow.

\subsection{Numerical Approximation of the PDE's}

It is well known that analytical solutions of PDE's can be obtained only under extremely special circumstances. For that reason, one usually resorts to finite dimensional representations of the underlying operators, by assuming that the solution of a PDE can be approximated by the sum of a certain number of basis 
functions [6]. It is obvious that if the basis functions individually satisfy the homogeneous boundary condition imposed on the function that we are after, then their sum will satisfy it as well. This is the first condition that each basis function is to fulfill. They also have to be easily computable and complete.

Despite the fact that there exist many basis functions satisfying these properties, the choice that is extremely suitable for a number of applications is either ordinary Fourier series or Chebyshev polynomials. The latter is the one that we will consider since it is extremely robust and gives good results in almost all situations [6].

The Chebyshev polynomial of degree $n$ is defined by

$$
T_{n}(\cos \theta):=\cos n \theta, \quad n=0,1,2, \ldots
$$

Based on this relationship, several Chebyshev polynomials can be obtained by introducing the replacement $y=\cos \theta$

$$
T_{0}(y)=1, \quad T_{1}(y)=y, \quad T_{2}(y)=2 y^{2}-1, \cdots .
$$

From the above it is obvious that $y \in[-1,1]$ and $\left|T_{n}(y)\right| \leq 1, \forall n=0,1,2, \ldots$ and $\forall y \in[-1,1]$. The Chebyshev polynomial of degree $n$ has exactly $n$ roots, $n$ maxima and $n$ minima. All the maxima and minima are equal to +1 and -1 , respectively.

Chebyshev polynomials can be defined alternatively by the three-term recurrence

$$
T_{n+1}(y)=2 y T_{n}(y)-T_{n-1}(y), \quad \forall n \geq 1,
$$

with $T_{0}(y)=1$ and $T_{1}(y)=y$. These relations play an invaluable role in the process of resorting to finite dimensional approximations of the operators. One more recursive relation deserves to be mentioned. It gives a connection between the $n$-th Chebyshev polynomial and the first derivatives, with respect to $y$, of the two adjacent polynomials

$$
T_{n}(y)=\frac{1}{2}\left(\frac{T_{n+1}^{\prime}(y)}{n+1}-\frac{T_{n-1}^{\prime}(y)}{n-1}\right), \quad \forall n \geq 2 .
$$

At first glance Chebyshev polynomials have a serious drawback: they are not orthogonal. This means that

$$
\int_{-1}^{1} T_{n}(y) T_{m}(y) d y \neq \delta_{n m} \alpha_{n}^{2}
$$

where $\delta_{n m}$ is the Kronecker delta function, and the constants $\left\{\alpha_{n}\right\}$ are the so-called normalization constants.

However, this problem can be overcome by defining the inner product in a slightly different manner. Suppose that the inner product is given by

$$
\left\langle T_{n}(y), T_{m}(y)\right\rangle=\int_{-1}^{1} T_{n}(y) T_{m}(y) \eta(y) d y,
$$

where $\eta(y)$ is a weight function. For $\eta(y)=\frac{1}{\sqrt{1-y^{2}}}$, the Chebyshev polynomials become orthogonal with respect to the inner product defined by (14). Mathematically expressed

$$
\left\langle T_{n}(y), T_{m}(y)\right\rangle=\left\{\begin{array}{cl}
\pi, & n=m=0 \\
\frac{\pi}{2}, & n=m \neq 0 \\
0, & n \neq m
\end{array}\right.
$$

The next natural step is to choose basis functions for the $\hat{v}$ and $\hat{\omega}_{y}$ bases. These functions have to satisfy boundary conditions given by (11). The most suitable choice in our case is the one that defines the $\hat{v}$ basis functions $\phi_{n}(y)$, and the $\hat{\omega}_{y}$ basis functions $\psi_{n}(y)$ in the following way

$\phi_{n}(y):=\left(1-y^{2}\right)^{2} T_{n}(y), \quad \psi_{n}(y):=\left(1-y^{2}\right) T_{n}(y)$.

All steps described above are necessary for the application of the Galerkin method. This method belongs to the class of the so-called spectral techniques. The main characteristic of spectral procedures is that the solution of a given equation is assumed to be represented as a sum of a finite number of basis functions. If we assume that the number of $\hat{v}$ and $\hat{\omega}_{y}$ basis functions is equal to $N$ and $M$, respectively, we can express $\hat{v}$ and $\hat{\omega}_{y}$ as

$$
\begin{aligned}
\hat{v}\left(k_{x}, y, k_{z}, t\right) & \approx \sum_{n=0}^{N} a_{n}\left(k_{x}, k_{z}, t\right) \phi_{n}(y), \\
\hat{\omega}_{y}\left(k_{x}, y, k_{z}, t\right) & \approx \sum_{n=0}^{M} b_{n}\left(k_{x}, k_{z}, t\right) \psi_{n}(y),
\end{aligned}
$$

where $a_{n}\left(k_{x}, k_{z}, t\right)$ and $b_{n}\left(k_{x}, k_{z}, t\right)$ are the so-called spectral coefficients. With a slight abuse of notation, we refer to them as $a_{n}(t)$ and $b_{n}(t)$, bearing in mind that they are functions of time parametrized by spatial frequencies $k_{x}$ and $k_{z}$. These coefficients are computed as a solution of a first order ODE obtained as a result of applying Galerkin method to equation (10). In order to get that ODE we have to find matrix representations of all operators entering (10) and to express $\hat{F}_{v}$ in terms of $\hat{v}$ basis functions. The last problem is the one that we describe here.

Since the forcing term is to be obtained as a function of $\phi_{n}$ 's, we have to approximate it in $y$ direction with something that is similar to a Dirac function. We choose $\hat{F}_{v}$ of the form

$\hat{F}_{v}=\delta(y+1-\varepsilon, t) \approx \hat{F}_{v y} \delta(t)=\exp \left\{-500(y+0.9)^{2}\right\} \delta(t)$.

Clearly, the shape of the disturbance is not an ideal impulse in the $y$ direction, but it is an approximation that is good enough to capture the phenomena we are interested in.

We shall continue by describing how one can find the representation of $\hat{F}_{v y}$ in terms of $\hat{v}$ basis functions. This problem amounts to finding coefficients $c_{n}$ in the following expression

$\hat{F}_{v y}=\sum_{n=0}^{N} c_{n} \phi_{n}(y)=\left[\begin{array}{lll}\phi_{0}(y) & \cdots & \phi_{N}(y)\end{array}\right]\left[\begin{array}{c}c_{0} \\ \vdots \\ c_{N}\end{array}\right]$.

This can be done by multiplying the last equation from the left hand side by $\left[\begin{array}{lll}\phi_{0}(y) & \cdots & \phi_{N}(y)\end{array}\right]^{\prime}$ and integrating from -1 to +1 , using the previously defined weight function $\eta(y)$. By doing so one can determine the vector $c$ as

$$
c=\Phi^{-1} l,
$$

where $\Phi$ is the $(N+1) \times(N+1)$ matrix whose $i j$-th element is determined by $\left\langle\phi_{i-1}, \phi_{j-1}\right\rangle=$ 
$\int_{-1}^{1} \phi_{i-1}(y) \phi_{j-1}(y) \eta(y) d y, \eta(y)=\frac{1}{\sqrt{1-y^{2}}}$, and $l$ is the $(N+1)$ vector with $l_{i}:=\left\langle\hat{F}_{v y}, \phi_{i-1}(y)\right\rangle$.

The second term on the right hand side of (18) is computed by performing numerical integration, while the first one can be determined without doing so by exploiting the fact that each $\phi_{n}(y)$ can be expressed as a linear combination of Chebyshev polynomials ${ }^{3}$. In that way, the $(N+1) \times(N+5)$ transformation matrix that maps the sequence of Chebyshev polynomials into the sequence of $\hat{v}$ basis functions, in the $N$-th order Galerkin approximation, is easily determined. Denoting that matrix by $P$, we can write

$$
\left[\begin{array}{c}
\phi_{0}(y) \\
\vdots \\
\phi_{N}(y)
\end{array}\right]=P\left[\begin{array}{c}
T_{0}(y) \\
\vdots \\
T_{N+4}(y)
\end{array}\right] .
$$

Thus, the matrix $\Phi$ is determined by

$$
\begin{aligned}
& \left\langle\left[\begin{array}{c}
\phi_{0}(y) \\
\vdots \\
\phi_{N}(y)
\end{array}\right],\left[\begin{array}{lll}
\phi_{0}(y) & \cdots & \phi_{N}(y)
\end{array}\right]\right\rangle \\
& =P\left\langle\left[\begin{array}{c}
T_{0}(y) \\
\vdots \\
T_{N+4}(y)
\end{array}\right],\left[\begin{array}{lll}
T_{0}(y) & \cdots & \left.T_{N+4}(y)\right]
\end{array}\right] P^{\prime}\right. \\
& =P G P^{\prime} \text {, }
\end{aligned}
$$

where $\langle\cdot, \cdot\rangle$ is the inner product defined by (14) and the $(N+5) \times(N+5)$ matrix $G$ is given by

$$
G=\operatorname{diag}\left\{\pi, \frac{\pi}{2}, \ldots, \frac{\pi}{2}\right\} .
$$

In a similar manner one can determine the matrix representations of all operators appearing in equation (10). It is important to notice that there is no need to perform numerical integration to do so, because of the appropriate choice of basis functions (15), and nice properties of the Chebyshev polynomials (12) and (13). In that way, the transformed model in the evolution form is converted into a set of first order ODE's, parametrized by $k_{x}$ and $k_{z}$, that has to be solved in order to obtain the values of the spectral coefficients $a_{n}(t)$ and $b_{n}(t)$. Numerical schemes that calculate the matrix representations of the underlying operators have been developed for the cases of both Couette and Poiseuille flow.

\subsection{Spatio-Temporal Impulse Response in the Case of Poiseuille Flow}

In this subsection we confine our attention to the analysis of results obtained by computing the impulse response of the Navier-Stokes equations linearized around a nominal velocity profile of the form $U(y)=1-y^{2}$. This velocity profile is an exact solution of the NavierStokes equations and the corresponding flow is usually referred to as Poiseuille flow.

\footnotetext{
${ }^{3}$ This can be done by using the definition of the $\hat{v}$ basis functions (15), and the three-term recurrence formula (12).
}

Since, the transformed evolution form model (9) is obtained by putting together linearized Navier-Stokes equations and the continuity equation in a suitable manner and then applying the Fourier transform in the $x$ and $z$ directions, it can be shown that Fourier pictures of the original streamwise and spanwise velocity perturbations can be extracted as functions of $\hat{v}, \hat{\omega}_{y}$, $k_{x}$ and $k_{z}$

$$
\begin{aligned}
\hat{u} & =\frac{i}{k_{x}^{2}+k_{z}^{2}}\left(k_{x} \partial_{y} \hat{v}-k_{z} \hat{\omega}_{y}\right), \\
\hat{w} & =\frac{i}{k_{x}^{2}+k_{z}^{2}}\left(k_{z} \partial_{y} \hat{v}+k_{x} \hat{\omega}_{y}\right) .
\end{aligned}
$$

Another quantity that deserves attention is streamwise vorticity whose definition, in the frequency domain, is given by

$$
\hat{\omega}_{x}:=\partial_{y} \hat{w}-i k_{z} \hat{v} .
$$

By combining (20) and (21) one can express $\hat{\omega}_{x}$ in terms of $\hat{v}$ and $\hat{\omega}_{y}$.

All simulations are done for $R=2000$, with $256 \times$ 256 grid points in the $k_{x}-k_{z}$ plane and $26 \hat{v}$ and $\hat{\omega}_{y}$ basis functions $(N=M=25)$. The ODE for spectral coefficients is solved using Matlab ${ }^{\circledR}$ 's function impulse.

Figure 2 shows pseudo-color plots of the streamwise velocity profile in the horizontal plane located in the vicinity of the upper wall at two different time instants: $T=150 \mathrm{~s}$ and $T=350 \mathrm{~s}$. Both plots have the rather characteristic arrowhead shape with streamwise streaky structures ubiquitous in both transitioning shear flows and fully turbulent boundary layers [7]. They also clearly illustrate the spanwise cross contamination of the turbulent spot and its growth as time progresses.
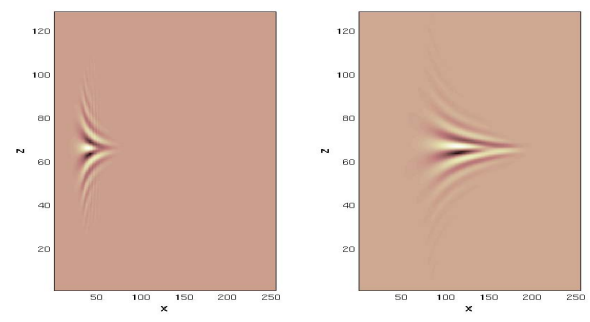

Figure 2: Streamwise velocity pseudo-color plots in the horizontal plane near upper wall $(y \approx$ $0.98)$ at $T=150 \mathrm{~s}$ and $T=350 \mathrm{~s}$.

The three dimensional isosurface plot of the streamwise velocity profile at $T=350 \mathrm{~s}$, illustrating high speed and low speed streaks, is shown in Figure 3. The alternating regions of high and low velocities are represented by two different colors: red represents high speed streaks, while green represents low speed streaks. One can notice two well developed regions almost symmetric with respect to the horizontal plane that divides the computational domain into two parts of equal size. The reason for their existence is shear which, in the case of Poiseuille flow, is equal to zero in the middle of the channel and grows linearly in the same fashion as one approaches either the lower or the upper wall. Since the evolution of $v$ is basically governed by a diffusion type equation, similar characteristics are not present in the 
isosurface plot of the wall-normal velocity. On the other hand, since both streamwise and spanwise velocities are functions of the wall-normal vorticity (20), and because the biggest energy growth is due to the coupling from $v$ to $\omega_{y}$, which in turn depends upon shear [5], the previously mentioned distinct regions characterize the evolution of both streamwise and spanwise velocity profiles.

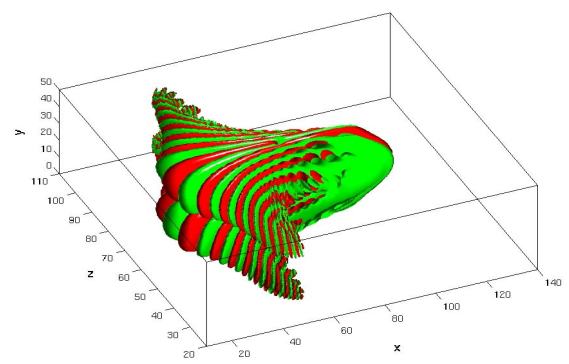

Figure 3: Streamwise velocity isosurface plot at $T=$ $350 \mathrm{~s}$. Red color represents high speed streaks, while green color represents low speed streaks.

Figure 4 shows simulation results illustrating pseudocolor plots of the streamwise velocity and vorticity profiles, respectively, in a cross section of the channel at $x=50$ (in terms of grid points). These plots further demonstrate the previously mentioned spanwise cross contamination and clearly show that the regions of high (warm colors) and low (cold colors) velocities occur in an alternating arrangement. Furthermore, it is apparent that there is a huge concentration of arrays of counter rotating streamwise vortices in the vicinity of the lower and upper walls. The relative position of vortices and streaks seems to agree qualitatively with experimentally observed data in which counter rotating vortices occur between high speed and low speed streaks. It is worth mentioning that, the wall normal coordinate in Figure 4 assumes values in the physical space, whereas the spanwise coordinate is given in terms of grid points.
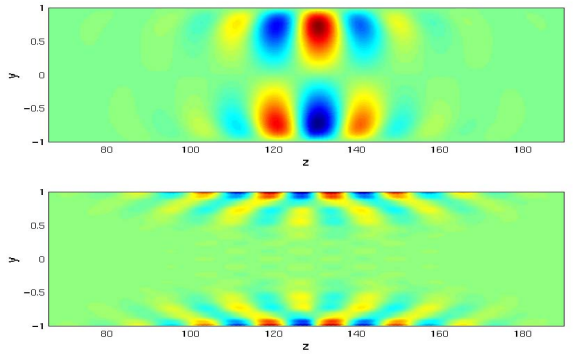

Figure 4: Pseudo-color plots of the streamwise velocity and vorticity profiles, respectively, at $x=50$ (in terms of grid points), and $T=270 \mathrm{~s}$.

All results shown in this subsection illustrate the ability of the linearized model subject to external forces to generate, at least qualitatively, structures (streamwise vortices and streaks) usually observed in real turbulent spots [7].

\section{Concluding Remarks}

This paper has dealt with the linearized Navier-Stokes equations subject to external forces, and it represents a further step towards their better understanding. We have derived a model in evolution form that incorporates the influence of external excitations in an exact manner. We have also developed the schemes for numerical simulation of our model in the cases of both Couette and Poiseuille flow. In particular, we have considered the situation in which the input signal assumes the form of a spatio-temporal impulse function entering the equation that governs the wall-normal velocity evolution. The reason for this choice of input signal is to see how changes in the wall-normal velocity would influence the evolution of the wall-normal vorticity through the coupling term which is responsible for large energy amplification [5].

Our results clearly demonstrate that streamwise elongated structures which alternate in the spanwise direction have been generated. These structures correspond to the so-called high and low speed streaks, which together with the vortices that counter-rotate between them are commonly observed in experiments investigating both transitional shear flows and fully turbulent boundary layers.

Since our ultimate objective is to control both the transition process and fully turbulent flows, current work can be described as a 'control oriented modeling'. This represents an important step because once the phenomena responsible for the transition to turbulence have been properly modeled and the control objective defined accordingly, controller design will be much more likely to give satisfactory results.

\section{References}

[1] L. N. Trefethen, A. E. Trefethen, S. C. Reddy, and T. A. Driscoll, "Hydrodynamic Stability Without Eigenvalues," Science, vol. 261, pp. 578-584, 30, July 1993.

[2] K. M. Butler and B. F. Farrell, "ThreeDimensional Optimal Perturbations in Viscous Shear Flow," Physics of Fluids A, vol. 4, p. 1637, 1992.

[3] L. H. Gustavsson, "Energy Growth of ThreeDimensional Disturbances in Plane Poiseuille Flow," J. Fluid Mech., vol. 98, p. 149, 1991.

[4] S. C. Reddy and D. S. Henningson, "Energy Growth in Viscous Channel Flows," J. Fluid Mech., vol. 252, pp. 209-238, 1993.

[5] B. Bamieh and M. Dahleh, "Energy Amplification in Channel Flows with Stochastic Excitation," to appear in Physics of Fluids, CCEC report 98-1012, University of California at Santa Barbara, http://www.engineering.ucsb.edu/ ${ }^{\sim}$ bamieh, 1998.

[6] J. P. Boyd, Chebyshev and Fourier Spectral Methods. Berlin, Heidelberg: Springer-Verlag, 1989.

[7] D. R. Carlson, S. E. Widnall, and M. F. Peeters, "A Flow-Visualization Study of Transition in Plane Poiseuille Flow," J. Fluid Mech., vol. 121, pp. 487-505, 1982. 